\title{
BMJ Open Efficacy of electroacupuncture for the treatment of constipation in Parkinson's disease: study protocol for a multicentre randomised controlled trial
}

\author{
Kunshan Li (D , ${ }^{1}$ Zhaoqin Wang, ${ }^{1}$ Yiyi Chen, ${ }^{2}$ Lirong Shen, ${ }^{3}$ Zhongqiu Li, ${ }^{1}$ \\ Yiwen Wu, ${ }^{4}$ Canxing Yuan, ${ }^{5}$ Yan Huang, ${ }^{6}$ Luyi Wu, ${ }^{1}$ Chunhui Bao, ${ }^{6}$ Wei Zhang, ${ }^{7}$ \\ Shifen $\mathrm{Xu}$ (1), ${ }^{8}$ Huangan $\mathrm{Wu}^{2}$
}

To cite: Li K, Wang Z, Chen Y, et al. Efficacy of electroacupuncture for the treatment of constipation in Parkinson's disease: study protocol for a multicentre randomised controlled trial. BMJ Open 2019;9:e029841. doi:10.1136/ bmjopen-2019-029841

- Prepublication history and additional material for this paper are available online. To view these files, please visit the journal online (http://dx.doi org/10.1136/bmjopen-2019029841).

$\mathrm{KL}$ and $\mathrm{ZW}$ contributed equally.

Received 14 February 2019 Revised 27 August 2019 Accepted 31 October 2019

Check for updates

C Author(s) (or their employer(s)) 2019. Re-use permitted under CC BY-NC. No commercial re-use. See rights and permissions. Published by BMJ.

For numbered affiliations see end of article.

Correspondence to Professor Huangan Wu; wuhuangan@126.com

Dr Shifen Xu; xu_teacher2006@126.com

\section{ABSTRACT}

Introduction Constipation is one of the most common nonmotor symptoms in Parkinson's disease (PD). Acupuncture can have a positive on chronic functional constipation and PD, but its efficacy for the treatment of constipation in PD has not yet been confirmed by high-quality clinical trials. Therefore, this study aims to evaluate the efficacy and safety of electroacupuncture (EA) in the treatment of constipation in PD.

Methods and analysis This study is a multicentre randomised controlled trial. A total of 124 qualified patients with PD and constipation will be randomly divided into the intervention group (62 participants will receive 12 weeks of $\mathrm{EA}$ +usual care) or the waitlist control group (62 participants will receive 12 weeks of usual care). EA will be performed three times per week from weeks 1-8, two times per week during weeks 9 and 10 , and once a week during weeks 11 and 12 . The primary outcome is the change in mean weekly spontaneous bowel movements from baseline to weeks 8 and 9 . The secondary outcomes are the changes from baseline in mean weekly bowel movements, mean weekly stool consistency, and mean weekly straining. Other secondary outcomes include the weekly doses of defecation drugs, Visual Analogue Scale for subjective improvements in stool symptoms, Unified Parkinson's Disease Rating Scale, and the time and number of steps required to walk $20 \mathrm{~m}$. Outcomes will be assessed at baseline, week 4, 8, 12 (intervention period); as well as at week 16, 24 (follow-up period).

Ethics and dissemination Ethical approval has been obtained from four local ethics committees. The results of the study will be published in peer-reviewed journals and will be disseminated through national and international conferences. Trial registration number ChiCTR1900021053

\section{INTRODUCTION}

With the increase in the percentage of ageing people in the global population, the incidence of Parkinson's disease (PD) is also increasing. The global prevalence rate of $\mathrm{PD}$ in the over 65 cohort is $1 \%-3 \% .{ }^{12}$ Previous studies have often focused on the management in motor symptoms of PD. However, non-motor
Strengths and limitations of this study

- This study will be one of the rare randomised controlled trials to assess the clinical efficacy of electroacupuncture (EA) in the treatment of constipation in Parkinson's disease (PD).

- In order to optimise the credibility of the research results, a multicentre trial will be utilised for this study.

- In addition to evaluating the clinical efficacy of EA in the treatment of constipation in $\mathrm{PD}$, this study will also investigate whether EA can play a role in maintenance therapy.

- This study will not include a sham EA group. Without blinding patients, the placebo effect of EA treatment cannot be ruled out.

symptoms (NMSs) have received increased attention in recent years. Studies have shown that some NMSs can occur earlier than motor symptoms, ${ }^{3}$ and can seriously affecting the patients' quality of life. Constipation is one of the most common and earliest NMSs, with a prevalence of up to $80 \%$ among patients with PD. ${ }^{4-6}$ Fifty percent of patients present constipation symptoms 10-20 years before the motor symptoms appear. ${ }^{7}$ Moreover, studies indicate that constipation may be one of the risk factors for PD because men or women afflicted with constipation (three or less bowel movements (BMs) per week) are two to five times more likely to be diagnosed with PD in the future compared with those who do not experience from constipation. ${ }^{8-10}$ Furthermore, common drugs used to treat PD, such as dopamine agonists, anticholinergics and catechol-oxylmethyltransferase inhibitors, can aggravate constipation symptoms.

The main cause of constipation in PD is slow colonic transit or pelvic floor and anal sphincter dysfunction. ${ }^{11}$ Therefore, the treatment of constipation in PD usually involves 


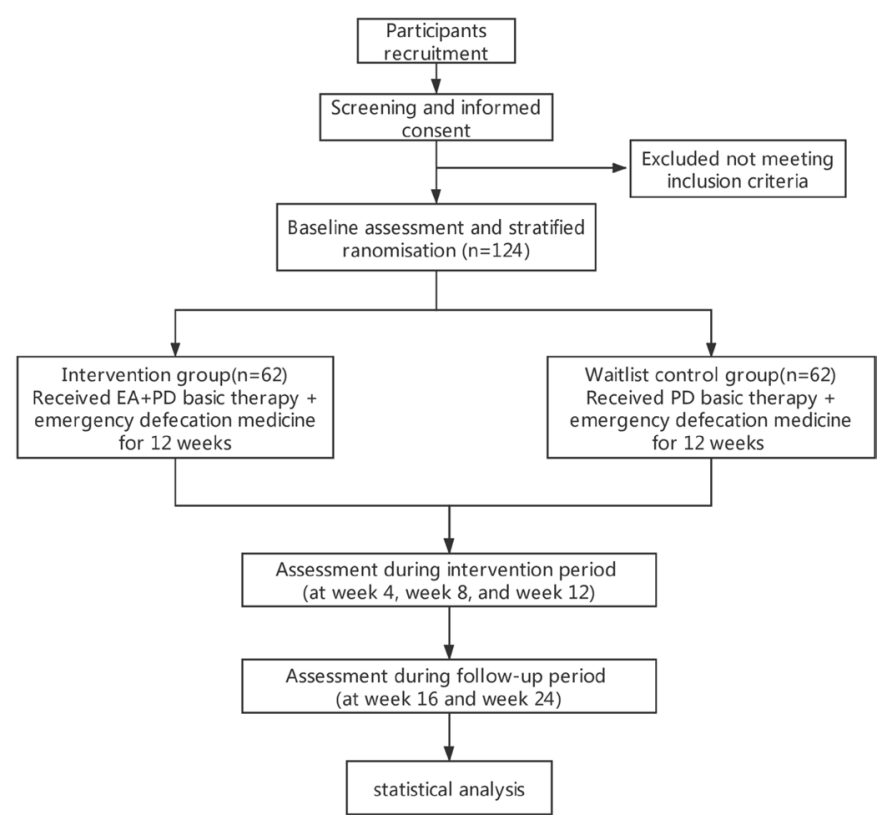

Figure 1 Flow chart. EA, electroacupuncture; PD, Parkinson's disease.

the use of laxative or prokinetic drugs. However, long-term treatment increases the likelihood of treatment-related adverse events (AEs). Approximately $50 \%$ of patients are unsatisfied with these drugs mainly due to their low efficacy, inconsistency or associated AEs. ${ }^{12}$

Acupuncture is an age-old practice in China, which has gained global popularity in recent decades. Studies have shown that acupuncture can increase stool frequency and improve stool consistency in functional constipation. ${ }^{13}{ }^{14} \mathrm{In}$ addition, acupuncture can delay the progression of PD to some extent. ${ }^{15} 16$ It can play an important role in reducing adverse reactions to medicine and improving NMSs. ${ }^{17-19}$ Nevertheless, we have not yet found a high-quality randomised controlled trial (RCT) study on acupuncture treatment for constipation in patients with PD.

Electroacupuncture (EA) is a form of acupuncture therapy that combines traditional acupuncture with electrical stimulation. The advantage of EA therapy is that the amount of stimulation can be objectively controlled, and the frequency and intensity of stimulation is replicable, ensuring that participants in clinical studies receive similar stimulation. In addition, as a form of acupuncture, EA is often used to treat constipation and PD. ${ }^{1415} 19$ Based on our previous studies, we have found that acupuncture treatment can ameliorate the symptoms of constipation in PD. ${ }^{20}$ Therefore, this study will investigate the efficacy of EA for treating constipation in PD through a multicentre RCT, in order to verify the feasibility and advantages of acupuncture treatment for constipation in PD.

\section{METHODS AND ANALYSIS Hypothesis}

Compared with usual care (UC) alone (PD basic therapy +emergency defecation medicine), EA+UCwill increase the frequency of defecation, ameliorate stool consistency and straining.

\section{Study design}

This study is a multicentre, parallel design RCT with blinded data collectors. It is estimated that 124 patients will be recruited and randomly divided into the intervention group $(\mathrm{EA}+\mathrm{UC})$ or the waitlist control group (UC alone) in a 1:1 ratio using stratified randomisation. The participants in the two groups will be treated for 12 weeks, and will then be followed up for 12 weeks. The flow chart of the trial process is shown in figure 1 . The research process and data collection are described in detail in table 1 and figure 2.

\section{Recruitment, setting and participants}

The trial will be conducted at Yueyang Hospital of Integrated Traditional Chinese and Western Medicine, Longhua Hospital, Shanghai Municipal Hospital of Traditional Chinese Medicine and Shuguang Hospital, all of which are affiliated with Shanghai University of Traditional Chinese Medicine. The participants will be recruited through the published advertisements in newspapers or by recruitment notices posted on the official network information platforms and the bulletin boards of each study site. Interested participants can contact the researchers through the provided telephone numbers. An independent researcher will conduct face-to-face interviews with the participants to explain the study, and those who volunteer to participate will be required to sign consent forms (see online supplementary file 1). After a baseline screening visit, participants, who meet the eligibility criteria, will be able to participate in the study.

\section{Inclusion criteria}

To be included in the trial, participants must (1) have a diagnosis of idiopathic PD according to the UK Parkinson's Disease Society Brain Bank Clinical Diagnostic Criteria $^{21}$; (2) meet the diagnostic criteria of Roman IV diagnostic criteria for functional constipation ${ }^{22}$; (3) be aged between 40 and 80 years; (4) have PD for $\geq 6$ months and constipation for $\geq 3$ months; (5) have mean weekly spontaneous BMs (SBMs) $<3$ times (based on the patient's -2 and 0 weeks stool diary record); (6) be unsatisfied with their current constipation treatment or have not yet received constipation treatment; (7) be in stages 1-4 of the Hoehn and Yahr scale and (8) be a voluntary participant in the study, agree to undergo relevant examinations and treatments, and sign the informed consent form.

\section{Exclusion criteria}

The exclusion criteria are as follows: (1) serious cardiovascular and cerebrovascular diseases, haematopoietic diseases, malignant tumours or other serious life-threatening diseases; (2) diagnosis of schizophrenia, major depression or cognitive impairment (cognitive impairment is defined based on the Mini-Mental State Examination (MMSE), which categorises cognitive 
Table 1 Schedule of enrolment, interventions and assessments

\begin{tabular}{|c|c|c|c|c|c|c|c|}
\hline \multirow{2}{*}{$\begin{array}{l}\text { Study period } \\
\text { Time point }\end{array}$} & \multicolumn{2}{|c|}{ Enrolment } & \multicolumn{3}{|c|}{ Intervention period } & \multicolumn{2}{|c|}{ Follow-up period } \\
\hline & Week -2 & 0-point & Week 4 & Week 8 & Week 12 & Week 16 & Week 24 \\
\hline Eligibility screening & $x$ & $x$ & & & & & \\
\hline Randomisation & & $x$ & & & & & \\
\hline \multicolumn{8}{|l|}{ Primary outcome } \\
\hline \multicolumn{8}{|l|}{ Secondary outcomes } \\
\hline Mean weekly BMs* & $\times$ & & $x$ & $x$ & $x$ & $x$ & $x$ \\
\hline Mean weekly stool consistency* & $x$ & & $x$ & $x$ & $x$ & $x$ & $x$ \\
\hline Mean weekly straining* & $x$ & & $x$ & $x$ & $x$ & $x$ & $x$ \\
\hline $\begin{array}{l}\text { Weekly emergency defecation drugs } \\
\text { usage* }^{*}\end{array}$ & $x$ & & $x$ & $x$ & $\times$ & $x$ & $x$ \\
\hline VAS & & $x$ & $x$ & $x$ & $x$ & $x$ & $x$ \\
\hline AEs & & & $x$ & $x$ & $x$ & $x$ & $x$ \\
\hline Compliance evaluation & & & $x$ & $x$ & $\times$ & $x$ & $x$ \\
\hline
\end{tabular}

*The results are based on the stool diary, which records the patient's stool for 2 weeks. Therefore, each stool diary recording time is $-2-0$ weeks, 4-5 weeks, 8-9 weeks, 12-13 weeks, 16-17 weeks and 23-24 weeks.

AEs, adverse events; BMs, bowel movements; SBMs, spontaneous bowel movements; UPDRS, Unified Parkinson's Disease Rating Scale; VAS, Visual Analogue Scale.

impairment based on different educational levels. The definitions of these criteria are as follows: people who are illiterate, have only received primary school education or have formal education higher than primary education will be classified as having cognitive impairment if their score less than 14, 20 or 24, respectively, on the MMSE); (3) history of laparotomy or anorectal surgery (except for haemorrhoid surgery, appendectomy in the last year or inguinal hernia repair) that may affect intestinal transit; (4) anal tumours, malformations, suspected intestinal obstruction or other organic diseases leading to constipation; (5) EA cannot be performed on acupuncture points due to skin diseases, limb defects or other conditions; (6) involvement in other clinical trials or having undergone acupuncture treatment within 30 days before participation, which may affect the results of this study; (7) acute gastrointestinal disease diagnosed within 2 weeks before treatment; (8) allergy to lactulose solution or glycerine enema and (9) pregnancy or lactation (under 60-year-old women will need to undergo a urine pregnancy test).

\section{Randomisation and allocation concealment}

Patients will be randomly assigned to the intervention group or the waitlist control group in a 1:1 ratio using stratified randomisation. The stratification will be based on two factors: (1) the severity of constipation (SBMs weekly=2 times or SBMs weekly $<2$ times); and (2) the study site. Independent statisticians will provide computergenerated random sequences and each stratum will be generated from a separate random sequence. Distribution will be conducted by a central web-based interactive randomisation service system. To prevent the researcher from predicting which group the next patient is allocated to, we will use the variable block randomisation method (block size of 4 or 6). When a participant is eligible, the researcher who conducted the random grouping will need to $\log$ onto the website to see the grouping situation of this participant.

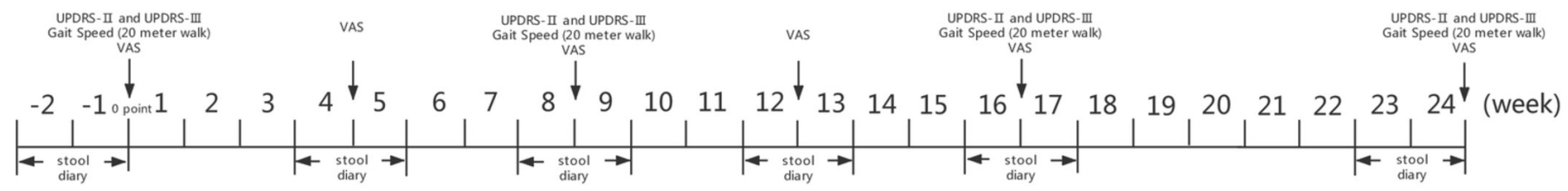

Figure 2 Outcome evaluation time point. UPDRS, Unified Parkinson's Disease Rating Scale; VAS, Visual Analogue Scale. 


\section{Blinding}

This trial is not blind to patients and acupuncturists, however, data collectors will be blinded. To improve blinding, each centre will have one acupuncturist and one data collector. The data collection location is independent of the treatment room. Patients and acupuncturists will be required to not disclose the patient's group allocation to the data collectors at any point during the trial. An independent statistician will conduct the statistical analyses of the results.

\section{Interventions}

During the trial, both groups will receive UC. In the intervention group, participants will receive EA during weeks 1-12. In the waitlist control group, participants have the option to receive 24 true acupuncture bonus sessions on conclusion of the study (ie, after week 24).

\section{UC treatments in both groups}

\section{Treatment of $P D$}

Considering the complexity of the clinical manifestations of PD and the principles of individualised treatment in PD clinical guidelines, the treatment for PD in this trial will not be strictly standardised. However, if the participant has received PD medication before enrolment, the dosage of the medicine should not be changed arbitrarily. The dose of different drugs can be converted to a total daily levodopa equivalent dose. ${ }^{23}$

\section{Emergency treatment for constipation}

If patients have no BMs for three or more consecutive days, they will be permitted to use an emergency treatment, which will be provided by the researchers. There are several options available to patients. For patients with no urge to defecate, it will be recommended that they first take oral lactulose solutions under the following protocol: $15 \mathrm{~mL}$ of oral lactulose solutions (oral lactulose solution, H20171057, Abbott Healthcare Products B.V. $15 \mathrm{~mL} /$ bag) taken at 12 hours intervals; if ineffective, $30 \mathrm{~mL}$ of oral lactulose solutions should be taken on the second day at 12 hours intervals. For patients who have an urge to defecate but are unable to pass stool, $20 \mathrm{~mL}$ of glycerine enemas (glycerine enema, H31021363, Shanghai Yunjia Huangpu Pharmaceutical Co. Ltd.; $20 \mathrm{~mL} /$ bottle) by rectal injection will be recommended. A combination of the two treatments will be recommended for patients who fail to respond to emergency treatment for 2 days. Patients will be advised not to use any other emergency treatments. Each use of emergency treatment will be recorded in the stool diary. If any other emergency treatment medication is used, this will also be recorded in the stool diary.

\section{EA add-on treatment in the intervention group}

Thirty EA sessions will be performed over a period of 12 weeks, with three sessions per week from weeks 1 to 8 , two sessions per week during weeks 9 and 10, and one session per week during weeks 11 and 12.
The acupuncturists who will provide treatment are the doctors with a qualification certificate in Traditional Chinese Medicine and at least 2 years of acupuncture experience. They are required to be registered with the Chinese Medical Doctor Association. All acupuncturists will receive one training session before commencing the study. Acupuncturists need to explain the EA treatment process to the patients and should describe any possible sensations during the treatment before starting the procedure.

Each patient in the intervention group will be treated at the bilateral acupoints of Connection Qianding (GV21) to Xuanlu (GB5), Connect Qianshencong (EX-HN1) to Xuanli (GB6), Tianshu (ST25), Fujie (SP14), Quchi (LI11), Hegu (LI4), Yanglingquan (GB34), Shangjuxu (ST37), Sanyinjiao (SP6), Zhaohai (KI6) and Taichong (LR3). When the patients are supine, they are to be treated with single-use stainless steel acupuncture needles (Hwato). Acupuncture on Tianshu (ST25) and Fujie (SP14) will be performed with $0.30 \times 50 \mathrm{~mm}$ or $0.30 \times 75$ $\mathrm{mm}$ needles (depending on the patient's body type), inserted slowly and vertically approximately $45-65 \mathrm{~mm}$ until the muscle layer of the abdominal wall is pierced. Acupuncture on the bilateral lines from Qianding (GV21) to Xuanlu (GB 5), the bilateral lines from Qianshencong (EX-HN1) to Xuanli (GB6) (for each line, two needles will be inserted), Hegu (LI4), Zhaohai (KI6) and Taichong (LR3) will be punctured with $0.25 \times 25 \mathrm{~mm}$ needles, inserted approximately 10-20 mm deep. Quchi (LI11), Yanglingquan (GB34), Shangjuxu (ST37) and Sanyinjiao (SP6) will be punctured using $0.25 \times 40 \mathrm{~mm}$ needles inserted approximately $30-35 \mathrm{~mm}$ deep. Manipulations such as twirling, lifting and thrusting will be performed to reach de qi, which is the sensation unique to acupuncture that includes soreness, heaviness and distension. Paired alligator clips from the EA instrument (SDZ-III EA device, Hua Tuo, Suzhou medical equipment) will be connected to Qianding (GV21) to Xuanlu (GB5; tremor type) or Connect Qianshencong (EX-HN1) to Xuanli (GB6; stiff type). In this case, either pairing may be selected according to the clinical manifestation of the patient's motor symptoms. The left acupoint is paired with the left acupoint, and the right acupoint is paired with the right acupoint. Finally, the bilateral points Tianshu (ST25) and Fujie (SP14) will be connected transversely to the EA instrument. The frequency of the wave is $10 / 50 \mathrm{~Hz} ; 10 \mathrm{~Hz}$ lasts for $5 \mathrm{~s}$, while $50 \mathrm{~Hz}$ lasts for $10 \mathrm{~s}$. The output pulse width is $0.2 \mathrm{~ms} \pm 30 \%$. The intensity of the current will be between 1 and $10 \mathrm{~mA}$, depending on the level the patient feels comfortable without feeling pain. EA treatment will last for $30 \mathrm{~min}$.

\section{Outcome measures and evaluation \\ Primary outcome}

The primary outcome is the change in mean weekly SBMs from baseline to weeks 8 and 9. SBM is defined as the number of times defecation has occurred in the past 24 hours without the use of emergency drugs or other 
methods to aid defecation. The data collectors will inform patients how to complete the stool diary.

\section{Secondary outcomes}

The secondary outcomes include the evaluation of constipation and PD with the following protocol: (1) assessment of constipation: changes from baseline in mean weekly BMs, mean weekly stool consistency (according to the Bristol Stool Form Scale score), and mean weekly straining score based on the stool diary; mean weekly frequency and mean dosage of emergency defecation drugs based on the stool diary; and use of Visual Analogue Scale (VAS) to measure improvements in the patient's subjective stool symptoms; (2) assessment of PD symptoms: sections II (motor experiences of daily living) and III (motor examination) of the Unified Parkinson's Disease Rating Scale (UPDRS) are used. If the patients appear 'on/off', the status of the patients in the 'on' stage is assessed. The time and number of steps required to walk $20 \mathrm{~m}$ (including step speed and average step distance) is also observed. Evaluation time points are shown in detail in figure 2.

\section{Safety assessment}

All patients will report any $\mathrm{AE}$ (described as unfavourable symptoms or diseases during the trial related to treatment of PD, emergency treatment for constipation and acupuncture) to the acupuncturists to avoid the data collectors becoming aware of the patient's group allocation. Common AEs related to acupuncture include fainting, local ecchymoses, continuous pain, acupoint location infection and dizziness, while AEs related to PD treatment include nausea, vomiting, hypotension, dry mouth and oedema. Common AEs related to emergency treatment for constipation include flatulence, abdominal pain and diarrhoea. The relationships between AEs and intervention methods will be assessed using the Standardised Case Causality Assessment. We ask patients in both groups to call acupuncturists to report any discomfort during the trial, and the acupuncturists need to ask in detail and determine their relevance to treatment. When AEs are identified as being associated with the intervention, it will be divided into general AEs or the other AEs. The other AEs may lead to stopping the intervention, such as fainting, acupoint location infection, vomiting, hypotension and diarrhoea occur. Acupuncturists should evaluate the patient's condition to decide whether the treatment can be continued if they happen. The results of AEs will be described as the number and proportion (\%) of AEs.

Any severe AE (SAE) will be determined in accordance with the International Conference on Harmonisation's harmonised tripartite guidelines ${ }^{24}$ and must be reported to the Independent Data and Safety Monitoring Board (DSMB).

\section{Data collection}

Each centre will have one data collector. Except for the stool diary, which will be completed by the patients, all efficacy indicators will be completed by the data collectors. All data collectors will receive training on how to administer the UPDRS and other scales.

\section{Data management}

Data entry procedure

The electronic data capture (EDC) system will be used for data entry and data management. Data entry will be carried out by one data collector who will be allocated to each centre. After clinical raw data collection has been completed, the data collectors will forward the data to the supervisor of the project for review. Error correction and data export will be conducted by the supervisor. After verification, the database will be locked and undergo statistical analysis. Participant information will be stored in the EDC system, which researchers can access using a password. The ethical review boards and DSMB shall have the right to consult the relevant records when necessary.

\section{Data interrogation procedure}

If the authenticity of the data is questioned, the data collector will be notified by the supervisor in the form of a data question form. The data collector's response will be entered into the data question form and returned to the statistician by the supervisor.

\section{Missing data procedure}

Missing data on the primary outcome will be imputed using the multiple imputation according to the missingat-random assumption. A control-based model will be used to evaluate the sensitivity to missing data departure from the assumption. The regression-based multiple imputation with baseline of mean weekly SBMs as fixed effect will be used. Multiple imputation will be used to impute missing data in full analysis set (FAS) and perprotocol set.

\section{Data monitoring}

This study established an independent DSMB, which is independent of the sponsor, consisting of an acupuncturist, a rehabilitation specialist and a statistician. The DSMB will supervises whether the trial follows the study design and standard guidelines, will monitor the progress of the trial, and will observe whether AEs and aetiologies are adequately recorded. Acupuncturists will need to assess, manage and classify AEs. In the event of a $\mathrm{SAE}$, it must be reported to the main researchers, ethics committees and the DSMB within 24 hours of the occurrence. The DSMB and the main researchers will discuss the issue. The ethics committees and the DSMB have the right to terminate the trial.

\section{Sample size}

We conducted a pilot trial to observe the effects of EA on patients with PD and constipation in Yueyang Hospital of Integrated Traditional Chinese and Western Medicine and Shanghai Municipal Hospital of Traditional Chinese Medicine. The results show that: an increase in mean weekly SBMs from baseline to week 8 and 9 was 0.925 , 
with an SD of 1.873 in the intervention group; an increase in mean weekly SBMs from baseline to week 8 and 9 was -0.323 , with an SD of 1.127 in the waitlist control group (see online supplementary file 2 ). The normality of the primary outcome in two groups were validated by ShapiroWilk test. We used two-sample t-test by power analysis and sample size software with a power of $90 \%$ and a twotailed significance level of 0.05 to determine the required sample size. For a more conservative estimate, we set the $\mathrm{SD}$ in both groups at 1.87. Assuming a $20 \%$ drop-out rate, the sample size will need to be to 62 per group, resulting in a total of 124 participants required for the study.

\section{Datasets included in the statistical analysis}

FAS with modified intention to treat

Patients in the intervention group will receive at least one treatment and have one therapeutic evaluation following treatment. Patients in the waitlist control group will receive at least two evaluations.

\section{Per protocol set}

Patients in this data set must meet the following criteria: (1) patients in both groups must complete the efficacy evaluation in week 12; (2) patients in the intervention group must reach a certain level of compliance, that is, patients must complete at least $80 \%$ of the 30 EA treatment sessions (ie, $\leq 6 \mathrm{EA}$ sessions may be missed).

\section{Safety set}

All patients will undergo at least one safety assessment.

\section{Statistical analysis}

The statistical analysis will be performed using SAS V.9.4 software with a two-tailed $\mathrm{p}<0.05$ considered statistically significant. Continuous data will be described in means and SD or median and interquartile ranges. Discrete data will be reported as a frequency and corresponding percentage.

The primary outcome is the change from baseline in mean weekly SBMs during weeks 8-9. The number of SBMs during weeks 8-9 and weeks $-2-0$ will be divided by two respectively to get the mean weekly number of SBMs. Then, the mean weekly of SBMs at weeks -2-0 will be subtracted from the mean weekly of SBMs at weeks 8-9. Analysis of the FAS and per-protocol set will be conducted simultaneously. We will assess the primary outcome by generalised linear model, including group, baseline SBMs and site as fixed effects and age, visit number, rescue medicine and other defecation aids as possible covariates. The result will be presented by $\mathrm{p}$ value and $95 \%$ CI.

Analysis of the secondary outcomes will be based on data from the FAS. The outcomes include (1) the changes from baseline in mean weekly BMs, mean weekly stool consistency (according to the Bristol Stool Form Scale score), and mean weekly straining score; (2) mean weekly frequency and mean dosage of emergency defecation drugs; (3) VAS score on subjective improvements in stool symptoms; (4) sections II and III of the UPDRS and (5) the time and number of steps required to walk 20 $\mathrm{m}$ (including step speed and average step distance). The same statistical methods used for the primary outcome will be used to analyse mean weekly BMs, mean weekly stool consistency, mean weekly straining score, step speed and average step distance, and UPDRS scores. In addition, the weekly mean dosage of emergency defecation drugs used will be compared between the two groups using an independent samples t-test or Wilcoxon rank-sum test and their mean frequencies will be compared using a $\mathrm{X}^{2}$ test or Fisher's exact test. VAS for stool symptoms will be compared between the two groups using Wilcoxon rank-sum test. The results will be presented by $\mathrm{p}$ value and $95 \%$ CI.

The safety evaluation will use data from the safety set group. AEs and SAEs will be compared between the two groups using a $\mathrm{X}^{2}$ test or Fisher's exact test.

\section{Patient and public involvement}

The patients and the general public were not involved in the design of this research.

\section{Ethics and dissemination}

Written informed consent will be obtained from all participants who agree to take part in this study. Any major modification of the protocol will be approved by the Ethical Review Board and the DSMB and will be documented on the Chinese Clinical Trials Registry.

During data collection, the data will be stored in a password-protected database. We plan to have the results of this study published in a peer-reviewed journal on time and disseminated through national and international conferences.

\section{DISCUSSION}

NMSs in PD are receiving increased attention from physicians and researchers. ${ }^{25-28}$ The efficacy of acupuncture in the treatment of NMSs in PD, such as sleep disorders and depression, has been supported by research. ${ }^{1829}$ However, there is still insufficient clinical research regarding use of acupuncture to treat constipation in PD. The efficacy of EA in the treatment of functional constipation has global value. ${ }^{19}$ Meanwhile, previous studies have shown that using acupuncture as an auxiliary therapy for PD can reduce toxicity and lead to increasing the efficacy of medication. ${ }^{29-31}$ Therefore, we hypothesised that EA therapy can ameliorate constipation in PD, although this hypothesis has not yet been supported by high-quality research. We designed this RCT to provide better evidence of the efficacy of EA for constipation in PD.

At present, treatments for constipation in PD mostly include oral medications, which can alleviate the clinical symptoms of functional constipation, but do not benefit PD itself. ${ }^{32-34}$ This study examined constipation and motor symptoms simultaneously because constipation in patients with PD is distinct from simple functional constipation owing to the interaction between parkinsonian motor symptoms and constipation. The aggravation 
of motor symptoms leads to a decrease in patients' daily activities and an increase in drug dosage, both of which may exacerbate constipation. Clinical studies have confirmed that severe constipation in patients with PD is caused by the loss of dopaminergic neurons in the intestinal muscles. ${ }^{35}$ We believe that treatment of PD may assist in reducing the loss of dopaminergic neurons in the body to a certain extent, which will be helpful in treating constipation. Meanwhile, constipation is also a risk factor for the development of PD. ${ }^{8-10}$ Chronic constipation leads to weakening gastrointestinal function, which may reduce the absorption of drugs and is consequently detrimental to the improvement of motor symptoms. If patients' motor symptoms worsen, they may need to increase the dosage of drugs, but some drugs can worsen the symptoms of constipation, which in turn creates a vicious cycle. To some extent, treating motor symptoms also helps to improve the symptoms of constipation.

Clinical trials of previous acupuncture treatments for PD have shown positive ${ }^{161736}$ and negative ${ }^{183738}$ results. On evaluation of the related articles, we suggest that the negative results may be related to the long intervals between acupuncture treatment and the short treatment period, which may have resulted in an insufficient dosage of acupuncture stimulation. Different amounts of stimulation may affect the efficacy of acupuncture, ${ }^{39}$ which may account for this inconsistency. ${ }^{40}$ Therefore, in order to obtain a more accurate result, the intervention group receiving three EA treatment sessions per week from weeks 1 to 8 . Maintenance treatments will be administered in the form of two sessions per week during weeks 9 and 10, and one session per week during weeks 11 and 12. Maintenance treatments serve two purposes. First, to observe whether reducing the treatment frequency can maintain the therapeutic effect after acupuncture has achieved a certain therapeutic threshold. This will prevent excessive treatment caused by increasing the number of patients' visits. Second, maintenance treatments are more economical. If a reduced frequency of acupuncture sessions remains effective as a maintenance treatment, the economic burden on patients can be reduced. The maintenance treatments are widely used in the current researches related to acupuncture. ${ }^{141}$

This study will not use a sham acupuncture group as a control group. The main reasons for this are as follows: (1) the primary outcome is the number of SBMs, which is objective and less susceptible to the placebo effect; (2) in the pilot study, we used a sham acupuncture treatment. The result indicates that patients in the sham acupuncture group struggled to attend the three treatment sessions per week, due to motor limitations and unconspicuous improvements in constipation symptoms. Therefore, the drop-out rate is high.

Due to the high frequency of visits and long-term treatment course in this study, patients will receive free EA treatment and emergency treatment for constipation throughout the trial to improve patient compliance. When they finish the relevant evaluation, they will receive a transport subsidy for each visit. On completing each evaluation over the 24-week trial, patients in the waitlist control group can also receive 24 true acupuncture sessions as a reward.

\section{TRIAL STATUS}

Patient recruitment will begin in August 2019 and is expected to finish at the end of 2022.

\section{Author affiliations}

${ }^{1}$ Shanghai University of Traditional Chinese Medicine, Shanghai, China

${ }^{2}$ Yueyang Hospital of Integrated Traditional Chinese and Western Medicine, Shanghai University of Traditional Chinese Medicine, Shanghai, China

${ }^{3}$ Shanghai Pudong New Area Hospital of Chinese Medicine, Shanghai, China ${ }^{4}$ Ruijin Hospital, Shanghai Jiao Tong University School of Medicine, Shanghai, China ${ }^{5}$ Longhua Hospital, Shanghai University of Traditional Chinese Medicine, Shanghai, China

${ }^{6}$ Key Laboratory of Acupuncture and Immunological Effects, Shanghai University of Traditional Chinese Medicine, Shanghai, China

${ }^{7}$ Departement of Biostatistics, School of Public Health, Fudan University, Shanghai, China

${ }^{8}$ Shanghai Municipal Hospital of Traditional Chinese Medicine, Shanghai University of Traditional Chinese Medicine, Shanghai, China

Acknowledgements We thank all participants and researchers at each subcentre for their contribution throughout all stages of this research.

Contributors $\mathrm{KL}$ and $\mathrm{ZW}$ are joint first authors. $\mathrm{KL}$ participated in the design of this study and drafted the manuscript. LW, CB and YH revised and edited this manuscript. WZ was responsible for the statistical plan and design. YC and ZL participated in the implementation of the project. ZW, LS, YW and CY will help recruit participants. SX contributed to the design of this study and critical revisions of the manuscript. HW conceived this research and is the research manager. All authors have read and approved this final manuscript.

Funding This work was supported by the Shanghai Science and Technology Committee (grant number: 18401970700), the National Key Basic Research Programme of China (grant number: 2015CB554501) and the Shanghai Municipal Commission of Health and Family Planning (grant number: 2018Y0143).

Competing interests None declared.

Patient consent for publication Not required.

Ethics approval This study protocol has been approved by four local ethics committees including those at: Yueyang Hospital of Integrated Traditional Chinese and Western Medicine (Approval No. 2018-121), Longhua Hospital (Approval No. 2019LCSY034), Shanghai Municipal Hospital of Traditional Chinese Medicine (Approval No. 2019SHL-KY-07-02), and Shuguang Hospital (Approval No. 2019676-31-01). This study conforms to the Declaration of Helsinki principles.

Provenance and peer review Not commissioned; externally peer reviewed.

Open access This is an open access article distributed in accordance with the Creative Commons Attribution Non Commercial (CC BY-NC 4.0) license, which permits others to distribute, remix, adapt, build upon this work non-commercially, and license their derivative works on different terms, provided the original work is properly cited, appropriate credit is given, any changes made indicated, and the use is non-commercial. See: http://creativecommons.org/licenses/by-nc/4.0/.

\section{ORCID iDs}

Kunshan Li http://orcid.org/0000-0002-0316-1753

Shifen Xu http://orcid.org/0000-0002-0173-5936

\section{REFERENCES}

1 Zaichick SV, McGrath KM, Caraveo G. The role of $\mathrm{Ca}^{2+}$ signaling in Parkinson's disease. Dis Model Mech 2017;10:519-35.

2 de Rijk MC, Launer LJ, Berger K, et al. Prevalence of Parkinson's disease in Europe: a collaborative study of population-based cohorts. neurologic diseases in the elderly Research Group. Neurology 2000;54:S21-3. 
3 Noyce AJ, Bestwick JP, Silveira-Moriyama L, et al. Meta-Analysis of early nonmotor features and risk factors for Parkinson disease. Ann Neurol 2012;72:893-901.

4 Barichella M, Cereda E, Pezzoli G. Major nutritional issues in the management of Parkinson's disease. Mov Disord 2009;24:1881-92.

5 Fasano A, Visanji NP, Liu LWC, et al. Gastrointestinal dysfunction in Parkinson's disease. Lancet Neurol 2015;14:625-39.

6 Berg D, Postuma RB, Adler CH, et al. Mds research criteria for prodromal Parkinson's disease. Mov Disord 2015;30:1600-11.

7 Chen Y, Yu M, Liu X, et al. Clinical characteristics and peripheral T cell subsets in Parkinson's disease patients with constipation. Int J Clin Exp Pathol 2015;8:2495-504.

8 Abbott RD, Petrovitch H, White LR, et al. Frequency of bowel movements and the future risk of Parkinson's disease. Neurology 2001:57:456-62.

9 Gao X, Chen H, Schwarzschild MA, et al. A prospective study of bowel movement frequency and risk of Parkinson's disease. Am J Epidemiol 2011;174:546-51.

10 Adams-Carr KL, Bestwick JP, Shribman S, et al. Constipation preceding Parkinson's disease: a systematic review and metaanalysis. J Neurol Neurosurg Psychiatry 2016;87:710-6.

11 Pfeiffer RF. Gastrointestinal dysfunction in Parkinson's disease. Semin Neurol 2011;17:10-15.

12 Johanson JF, Kralstein J. Chronic constipation: a survey of the patient perspective. Aliment Pharm Ther 2010;25:599-608.

13 Zheng H, Liu Z-S, Zhang W, et al. Acupuncture for patients with chronic functional constipation: a randomized controlled trial. Neurogastroenterol Motil 2018;30:e13307.

14 Liu Z, Yan S, Wu J, et al. Acupuncture for chronic severe functional constipation: a randomized trial. Ann Intern Med 2016;165:761-9.

15 Lei H, Toosizadeh N, Schwenk M, et al. A pilot clinical trial to objectively assess the efficacy of electroacupuncture on gait in patients with Parkinson's disease using body worn sensors. PLoS One 2016;11:e0155613.

16 Doo K-H, Lee J-H, Cho S-Y, et al. A prospective open-label study of combined treatment for idiopathic Parkinson's disease using acupuncture and bee venom acupuncture as an adjunctive treatment. J Altern Complement Med 2015;21:598-603.

17 Fukuda S, Kuriyama N, Tsuru $\mathrm{H}$, et al. Immediate effects of acupuncture on tongue pressure including swallowing reflex latency in Parkinson's disease. Acupunct Med 2016;34:59-61.

18 Zeng B-Y, Zhao K. Effect of acupuncture on the motor and nonmotor symptoms in Parkinson's disease-a review of clinical studies. CNS Neurosci Ther 2016;22:333-41.

19 Wang F, Sun L, Zhang X-zhe, et al. Effect and Potential Mechanism of Electroacupuncture Add-On Treatment in Patients with Parkinson's Disease. Evid Based Complement Alternat Med 2015;2015:1-11.

20 Shen LR, Wang QD, Shen LP, et al. Therapeutic observation of acupuncture-moxibustion plus MA ren capsules for constipation in middle-late stage Parkinson disease. Shanghai Journal of Acupuncture and Moxibustion 2018;37:1381-5.

21 Rajput DR. Accuracy of clinical diagnosis of idiopathic Parkinson's disease. J Neurol Neurosurg Psychiatry 1993;56:938-9.

22 Rome Foundation. Rome IV diagnostic criteria for functional constipation, 2016. Available: www.nature.com/nrgastro/journal/v13/ n9/extref/nrgastro.2016.110-s1.pdf

23 Tomlinson CL, Stowe R, Patel S, et al. Systematic review of levodopa dose equivalency reporting in Parkinson's disease. Mov Disord 2010;25:2649-53.
24 International Conference on Harmonisation of Techinical Requirements for Registration of Pharmaceuticals for Human Use. Ich harmonised tripartite guideline: safety pharmacology studiesfor human pharmaceuticals S7A. Available: http://www.ich.org/fileadmin/ Public_Web_Site/ICH_Products/Guidelines/Safety/S7A/Step4/S7A_ Guideline.pdf

25 Pfeiffer RF. Non-Motor symptoms in Parkinson's disease. Parkinsonism Relat Disord 2016;22:S119-22.

26 Song R, Grabowska W, Park M, et al. The impact of tai chi and Qigong Mind-body exercises on motor and non-motor function and quality of life in Parkinson's disease: a systematic review and metaanalysis. Parkinsonism Relat Disord 2017;41:3-13.

27 O'Brien C, Clemson L, Canning CG. Multiple factors, including non-motor impairments, influence decision making with regard to exercise participation in Parkinson's disease: a qualitative enquiry. Disabil Rehabil 2016;38:472-81.

28 Fox SH, Regina K, Shen-Yang L, et al. The movement disorder society evidence-based medicine review update: treatments for the motor symptoms of Parkinson's disease. Mov Disord 2011;26:S2-41.

29 Zhuang X, Wang L. Acupuncture treatment of Parkinson's disease--a report of 29 cases. J Tradit Chin Med 2000;20:265-7.

30 Chen F-P, Chang C-M, Shiu J-H, et al. A clinical study of integrating acupuncture and Western medicine in treating patients with Parkinson's disease. Am J Chin Med 2015;43:407-23.

31 Lee S-H, Lim S. Clinical effectiveness of acupuncture on Parkinson disease: a PRISMA-compliant systematic review and meta-analysis. Medicine 2017;96:e5836.

32 Parkinson Study Group. Electronic address: pfeiffro@ohsu.edu. A randomized trial of relamorelin for constipation in Parkinson's disease (MOVE-PD): trial results and lessons learned. Parkinsonism Relat Disord 2017;37:101-5.

33 Barichella M, Pacchetti C, Bolliri C, et al. Probiotics and prebiotic fiber for constipation associated with Parkinson disease: an RCT. Neurology 2016;87:1274-80.

34 Ondo WG, Kenney C, Sullivan K, et al. Placebo-Controlled trial of lubiprostone for constipation associated with Parkinson disease. Neurology 2012;78:1650-4.

35 Singaram C, Gaumnitz EA, Torbey C, et al. Dopaminergic defect of enteric nervous system in Parkinson's disease patients with chronic constipation. Lancet 1995;346:861-4.

36 Seung-Yeon C, So-Ra S, Hak Young R, et al. Effectiveness of acupuncture and bee venom acupuncture in idiopathic Parkinson's disease. Parkinsonism Relat Disord 2013;333:e115-e15.

37 Cristian A, Katz M, Cutrone E, et al. Evaluation of acupuncture in the treatment of Parkinson's disease: a double-blind pilot study. Mov Disord 2005;20:1185-8.

38 Kluger BM, Rakowski D, Christian M, et al. Randomized, controlled trial of acupuncture for fatigue in Parkinson's disease. Mov Disord 2016;31:1027-32.

39 Sjölund BH. Acupuncture or acupuncture? Pain 2005;114:311-2.

40 Meng $\mathrm{ZH}$. The quantity-effect relationship of acupuncture. World Chinese Medicine 2014;12:1581-5.

41 Hershman DL, Unger JM, Greenlee $\mathrm{H}$, et al. Effect of acupuncture vs sham acupuncture or Waitlist control on joint pain related to aromatase inhibitors among women with early-stage breast cancer: a randomized clinical trial. JAMA 2018;320:167-76. 